\section{Commentary: The aortic valve after rheumatic mitral valve surgery. Remarkably stable!}

\author{
Manuel J. Antunes, MD, PhD, DSc
}

Acute rheumatic fever is still endemic in most low- and middle-income countries, and rheumatic heart valve disease, one of the most important causes of mortality, is especially prevalent in young patients, with a mean age of 20 to 25 years in patients who reach surgery. The disease is much rarer in developed countries of the Western world and some parts of Asia, but in these regions, there remain many patients who have late consequences of the acute phase of rheumatic fever that occurred in their infancy or youth 4 or 5 decades ago. These 2 stages of the disease are different in their pathology and evolution and must not be compared. In either case, multiple valve involvement is common, especially the mitral valve (MV) and aortic valve (AV), and this often generates a decision problem with regards to which valve(s) to intervene during the surgery. It has been reported that $2 \%$ of patients presenting with mild AV disease (AVD) at the time of MV replacement showed significant progression to require AV replacement (AVR) during a long follow-up. ${ }^{1}$

In this issue of the Journal, Kim and colleagues ${ }^{2}$ from Seoul, Korea, analyze the evolution of the native AV function by the progression of rheumatic disease in 1155 consecutive patients who underwent first-time isolated rheumatic MV surgery in their center from 1997 to 2015, with a median follow-up of almost 12 years. Freedom from the primary end point (composite of progression to severe AV dysfunction [AVD] or a requirement of subsequent AVR during follow-up) at 10 years was $97 \%$ for the whole

\footnotetext{
From the Clinic of Cardiothoracic Surgery, Faculty of Medicine, University of Coimbra, Coimbra, Portugal.

Disclosures: The author reported no conflicts of interest.

The Journal policy requires editors and reviewers to disclose conflicts of interest and to decline handling or reviewing manuscripts for which they may have a conflict of interest. The editors and reviewers of this article have no conflicts of interest.

Received for publication March 14, 2021; revisions received March 14, 2021; accepted for publication March 15, 2021; available ahead of print March 18, 2021. Address for reprints: Manuel J. Antunes, MD, PhD, DSc, Faculty of Medicine, University of Coimbra, 3000-548 Coimbra, Portugal (E-mail: mjantunes@fmed.uc.pt). J Thorac Cardiovasc Surg 2023;165:630-1

$0022-5223 / \$ 36.00$

Copyright (C) 2021 by The American Association for Thoracic Surgery

https://doi.org/10.1016/j.jtcvs.2021.03.048
}

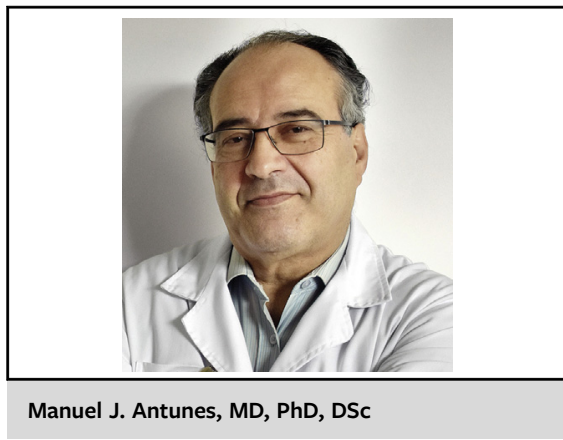

CENTRAL MESSAGE

The association of rheumatic mitral valve (MV) and aortic valve (AV) disease, in different degrees, is frequent. However, the untouched AV remains remarkably stable for long periods after MV surgery!

group and $92 \%$ for patients with mild or greater AV dysfunction at the time of MV surgery. Hence, the authors concluded that "progression of severe AVD and the need for AVR are uncommon in patients undergoing rheumatic MV surgery" but caution that "such events were relatively common among those with $\geq$ mild AV dysfunction at the time of MV surgery."

This is probably the largest such series with a long follow-up, and the data from this paper may help in the decision-making process. Although the conclusion was not unexpected, as we all know the progressive nature of rheumatic heart valve disease, the recommendation that "concomitant AV surgery during rheumatic MV surgery may offer clinical benefits in patients with mild-tomoderate AV dysfunction (to be confirmed by further comparative studies)" is, in my opinion, exaggerated for several reasons. First, an extra 10 or 12 years without an aortic prosthesis is a significant gain, especially in younger patients, keeping in mind the incidence of prosthetic-related adverse events, which were not analyzed in this study. Second, worsening of baseline AV dysfunction more than moderate was detected in $81(0.98 \%$ /patient-year $)$ patients, but only 26 fulfilled the primary end point, which is really not a very large number to justify such statement. Third, with the advent of percutaneous AV intervention, an early AVR may 
not be justified. Curiously, the authors do not appear to have a great preoccupation about this, judging by the fact that only less than $20 \%$ of the MVs were repaired.

Classically, the guidelines for management of patients with valvular heart disease recommend that AVR is reasonable in patients with moderate AVD who undergo other cardiac surgery (Class IIa recommendation). ${ }^{3}$ Hence, the question here lies only on what to do with mild AVD. This is a point that is not clarified by the current study. $\mathrm{AV}$ dysfunction none-to-trivial was present in $76.2 \%$ and $70.1 \%$ and mild in $22.2 \%$ and $21.8 \%$, respectively, before and after. The differences are not large, leaving $4.5 \%$ of patients whose AV dysfunction increased to moderate and $2.1 \%$ whose dysfunction increased to severe. In the final analysis, the latter are the ones who might have been affected by the initial "respect" the AV policy. Not bad at all! In this series, it would have taken 228 early AVRs to prevent the 14 events of late severe AVD that required AVR. Furthermore, the "rapid AV dysfunction observed in patients with $\geq$ mild AV dysfunction at baseline" was only evident after 10 years of follow-up. Risk factors for developing severe AV deterioration were identifiedexpectedly, AV peak pressure gradient, and degree of aortic regurgitation, most importantly the former. Isn't this the most important in deciding who should have AV replacement in the first place?

Again, and most importantly, this paper deals with a population with a mean age of 52 years, which is characteristic of the rheumatic population of the authors' country. It cannot be compared with the populations of low- and middle-income countries, to which the authors refer in their introduction, usually with a mean age in the 20 s, where the consequences of an added prosthesis are far greater. In these patients, the progression of AVD is more frequent and faster because the inflammatory rheumatic process has not settled yet. From this point of view, the first paragraph of the introduction of this paper by Kim and colleagues is susceptible to generating some confusion.

Finally, the authors comment on the type of prosthesis used: "if a tissue valve is chosen (for replacement of the $\mathrm{MV}$ ), it would be reasonable to defer the AV surgery and address it at the second reoperation as needed during follow-up." Rather, shouldn't the perspective of progression of the AVD increase the awareness and/or willingness to repair the MV, leaving bivalvular replacement for a later stage? Many recent papers coming from the same area of the globe, including at least one originating from the same group, ${ }^{4}$ attest to the long-term efficacy of rheumatic mitral valvuloplasty. In this regard, the controversy goes on. 5

In conclusion, the paper by Kim and colleagues adds further to our knowledge on the management of complex rheumatic valvular disease, and the authors are to be congratulated for their persistent work in this field. However, the final suggestion that "AV surgery during rheumatic MV surgery may offer clinical benefits in patients with mild-to-moderate AV dysfunction" is, in my view, not supported by the data produced. As they say, only "further comparative studies can determine that." In the meantime, we can only say that the AV remains remarkably stable after rheumatic MV surgery!

\section{References}

1. Vaturi M, Porter A, Adler Y, Shapira Y, Sahar G, Vidne B, et al. The natural history of aortic valve disease after mitral valve surgery. J Am Coll Cardiol. 1999;33:2003-8.

2. Kim HR, Kim WK, Kim HJ, Kim JB, Jung SH, Choo SJ, et al. The fate of aortic valve after rheumatic mitral valve surgery. J Thorac Cardiovasc Surg. 2023;165: 622-9.e2.

3. Nishimura RA, Otto CM, Bonow RO, Carabello BA, Erwin JP III, Guyton RA, et al. Valvular heart disease management guideline executive summary. J Am Coll Cardiol. 2014;63:2438-88.

4. Kim WK, Kim HJ, Kim JB, Jung SH, Choo SJ, Chung CH, et al. Clinical outcomes in 1731 patients undergoing mitral valve surgery for rheumatic valve disease. Heart. 2018;104:841-8

5. Antunes MJ. Repair for rheumatic mitral valve disease. The controversy goes on! Heart. 2018;104:796-7. 